\title{
The GRACE Scale in the Prognosis of Patients with Takotsubo Syndrome
}

\author{
Malgorzata Zalewska-Adamiec $\mathbb{D}^{1},{ }^{1}$ Lukasz Kuzma, ${ }^{1}$ Slawomir Dobrzycki, ${ }^{1}$ \\ and Hanna Bachorzewska-Gajewska ${ }^{1,2}$ \\ ${ }^{1}$ Department of Invasive Cardiology, Medical University of Bialystok, Białystok, Poland \\ ${ }^{2}$ Department of Clinical Medicine, Medical University of Bialystok, Białystok, Poland \\ Correspondence should be addressed to Malgorzata Zalewska-Adamiec; mzalewska5@wp.pl
}

Received 1 January 2020; Revised 17 March 2020; Accepted 9 April 2020; Published 25 April 2020

Academic Editor: Jochen Wöhrle

Copyright (c) 2020 Malgorzata Zalewska-Adamiec et al. This is an open access article distributed under the Creative Commons Attribution License, which permits unrestricted use, distribution, and reproduction in any medium, provided the original work is properly cited.

Background. The prognosis of Takotsubo syndrome (TTS) is comparable to that of the non-ST-elevation myocardial infarction (NSTEMI). The GRACE scale is used to assess the risk of premature and long-term mortality in patients with NSTEMI in order to select the most favorable treatment strategy. Methods. 101 patients with TTS hospitalized in four centers of invasive cardiology in Podlaskie Voivodeship during the period 2008-2012 were included in the study. The patients were divided into two groups: I-52 patients (GRACE $\leq 140$ points) and II-49 patients (GRACE $>140$ points). Results. The mean GRACE score in the study group was 138.66. The in-hospital stay of Takotsubo in the patients with higher GRACE scores was associated with higher incidence of pneumonia ( $36.7 \%$ vs $7.69 \%, p=0.0004)$, rhythm abnormalities $(17.3 \%$ vs $3.85 \%, p=0.026)$, and serious complications (cardiogenic shock, pulmonary edema, and sudden cardiac arrest) (30.6\% vs 5.77\%, $p=0.001)$. The mean observation period was 7.2 years. A significantly higher risk of 6 -month $(18.37 \%$ vs $3.85 \%, p=0.019)$, 1 -year $(22.45$ vs $3.85 \%, p=0.005), 3$-year $(40.82$ vs $3.85 \%, p<0.0001)$, 5 -year (42.86\% vs 3.85\%, $p<0.0001$ ), and 7 -year mortalities (53.06\% vs $9.62 \%, p<0.0001$ ) was observed in the group of patients with a GRACE score $\geq 140$. At multivariate analysis including low BMI, low eGFR, and a higher GRACE score, all these factors were independent predictor of death $(p=0.042 ; p=0.010 ; p=0.041)$. The ROC curve presents the discriminatory scores of the GRACE scale for the follow-up prognostication. The area under ROC curve (AUC) for the GRACE scale was 0.805 (95\% CI: $0.718-0.892, p<0.0001$ ), with a cut-off value of 153 points, sensitivity of $74 \%$, and specificity of $77 \%$ for TTS. Conclusion. The GRACE scale is highly valuable for the prognostication of death risk in patients with TTS in the early and long-term observation.

\section{Introduction}

Takotsubo syndrome (TTS) consists in the disorders related to the intermittent contractility of left ventricular wall induced by stress, with a significant increase of cardiac enzymes, ischemic changes in electrocardiogram (ECG) records, and lack of significant coronary artery stenosis. TTS is relatively rare and diagnosed in approximately $1-2 \%$ of the patients hospitalized for myocardial infarction $[1,2]$.

For years, TTS was considered a benign disease; however, numerous case reports and registry studies have reported the possibility of highly severe complications, such as severe heart failure, rhythm abnormalities including sudden cardiac arrest and cardiac rupture, in the acute phase of the disease. Takotsubo may relapse in the follow-up period, and the prognosis is comparable to that of the non-ST-elevation myocardial infarction (NSTEMI) [3-6].

Scales to assess prognosis and to facilitate the qualification of patient for various therapeutic methods have been developed for many disease entities [7-9]. In the case of Takotsubo, so far only the TTS diagnosis scale, InterTAK (InterTAK Diagnostic Score) [10], has been developed, while there is no scale dedicated to the assessment of the prognosis of Takotsubo patients. The GRACE scale (Global Registry of Acute Coronary Events) has been used for years in patients with NSTEMI in order to select the most favorable treatment 
strategy and to assess the risk of death in long-term followup $[11,12]$. As the clinical presentation of TTS is similar to that of acute coronary syndromes and the long-term prognosis is comparable to NSTEMI, a possibility exists that the GRACE may be helpful in the assessment of Takotsubo prognosis.

The study objective was to verify if the GRACE scale can be applied for the assessment of early and long-term deaths in Takotsubo patients.

\section{Methods}

2.1. Study Population. A total of 101 patients with TTS were hospitalized in four centers for invasive cardiology in Podlaskie Voivodeship during the period 2008-2012. TTS was diagnosed based on the applicable Mayo Clinic criteria [13], including 6 patients who were diagnosed with TTS despite the presence of significant changes in the coronary arteries. The new Takotsubo criteria contained in the position of the European Society of Cardiology [14]; it is possible to diagnose TTS with the presence of significant coronary stenosis; therefore, all 101 patients were included in the study.

The GRACE scores were calculated for all patients taking into account the age, heart rate, systolic blood pressure, plasma creatinine concentration, Killip-Kimball class at admission, cardiac arrest at admission, ST-segment changes, and elevated myocardial necrosis markers. Scores on the GRACE 2.0 scale and the risk of in-hospital, 6-month, 1year, and 3-year death were estimated using the calculator available on the http://www.gracescore.org website.

The patients were divided into 2 groups depending on the score on the GRACE scale as follows: Group I-52 patients (GRACE score $\leq 140$ points) and Group II-49 patients (GRACE score $>140$ points).

2.2. Follow-Up Examination. A long-term observational study after 7 years was conducted based on data concerning deaths obtained from the Division of Data Sharing of the Ministry of Digitalization (Department of National Systems). Mortality due to any cause was assumed as the main endpoint of the study.

2.3. Statistical Analysis. The obtained data were subjected to statistical analysis. The quantitative data were compared by Student's test and the Mann-Whitney $U$ test, whereas qualitative data were compared by the ch2 test and Fisher's test. The sensitivity and specificity of the Grace scale were assessed using the ROC curve (Receiver Operating Characteristic curve). The survival analysis was conducted with the Kaplan-Meier method, and the comparison of groups with the log-rank test. The multivariate analysis was conducted with the logistic regression method. Aside from the factors in which the tested groups differed significantly, the analysis also included the factors of potential significance in the prognosis of patients with acute coronary syndromes. The value of $<0.05$ was assumed as statistically significant.
The statistical analysis was performed using the STATISTICA 13.1 software.

The study was carried out upon approval from the Bioethics Committee of the Medical University of Bialystok.

\section{Results}

3.1. Studied Population and Group Comparison. Table 1 presents the characteristics of the entire Takotsubo group (101 patients) and the study groups with lower and higher GRACE scores. The study group comprised $87 \%$ of women, and the mean age of patients was 68 . The mean GRACE score was 138.66 (SD-36.75), while the median was -138 .

Patients with a GRACE score $>140$ were older and had a lower body mass index (BMI). They had a higher incidence of arterial hypertension, diabetes, chronic obstructive pulmonary disease (COPD), cancer, and chronic kidney disease, in addition to lipid disorders and a burdened family history.

Patients with a higher GRACE had significantly lower left ventricular ejection fraction (LVEF). Furthermore, in these patients, cardiac catheterization more frequently demonstrated atherosclerotic changes in the coronary arteries (irrelevant and significant), while the ECG records more frequently demonstrated ST-segment elevation.

In the group of patients with a GRACE score $>140$, the results of the laboratory tests demonstrated higher values of inflammatory parameters and higher levels of cardiac enzymes, glucose on admission, and creatinine, whereas the concentrations of all lipid fractions were lower.

In the group with a GRACE score $\geq 140$, significantly lower values of the systolic arterial pressure and higher heart rates were observed on admission. The in-hospital stay of the Takotsubo patients with higher GRACE scores was associated with higher incidence of pneumonia, rhythm abnormalities, cardiogenic shock, pulmonary edema, and sudden cardiac arrest. Cardiac rupture was noted only in this group, in 3 patients.

3.2. GRACE Scale and Mortality. In the long-term study, the mean observation period was 87 months ( 7.2 years). The risk of death in 1-year and 3-year follow-up periods estimated using the GRACE calculator was higher in the group of patients with higher GRACE scores. In the observational study, a significantly higher risk of 6-month and 1-, 3-, 5-, and 7-year mortality was observed in the group of patients with a GRACE score $\geq 140$. Data on mortality and the Kaplan-Meier curves are presented in the table (Table 2) and in the figure (Figure 1).

In the multivariate analysis performed by the logistic regression method, the risk factors for death in long-term follow-up are low BMI $\left(<20 \mathrm{~kg} / \mathrm{m}^{2}\right)$, low GFR $(<60 \mathrm{~m} / \mathrm{min} /$ $1.72 \mathrm{~m}^{2}$ ), and more points on the GRACE scale (Table 3 ).

3.3. Predictive Value of the GRACE Scale. In Figure 2, the ROC curve presents the discriminatory scores of the GRACE scale in the predicting long-term prognosis. The area under ROC curve (AUC) for the GRACE scale was $0.805(95 \%$ confidence interval [CI] 0.718-0.892, $p<0.001$ ), with a cut- 
TABLE 1: Results-comparison of 2 groups of patients with Takotsubo $(N=101)$.

\begin{tabular}{|c|c|c|c|c|}
\hline GRACE & $\begin{array}{c}\text { TTS } N=101(\mathrm{SD}) \\
N(\%)\end{array}$ & $\begin{array}{c}\mathrm{TTS} \leq 140 N=52(\mathrm{SD}) \\
N(\%)\end{array}$ & $\begin{array}{c}\mathrm{TTS}>140 N=49(\mathrm{SD}) \\
N(\%)\end{array}$ & $p$ \\
\hline \multicolumn{5}{|l|}{ Clinical and demographic characteristics: } \\
\hline Age (years) ${ }^{\text {GRACE }}$ & $68.01(13.88)$ & $58.62(12.62)$ & $77.98(6.26)$ & $<0.0001$ \\
\hline \multirow{2}{*}{ Female sex (\%) } & 88 & 45 & 43 & \multirow{2}{*}{0.855} \\
\hline & $87.13 \%$ & $86.54 \%$ & $87.76 \%$ & \\
\hline Body mass index (BMI) kg/m ${ }^{2}$ & $25.79(4.83)$ & $26.88(4.54)$ & $24.69(4.97)$ & 0.038 \\
\hline \multirow{2}{*}{ History of malignancy (\%) } & 5 & 0 & 5 & \multirow{2}{*}{0.018} \\
\hline & $4.95 \%$ & $0.0 \%$ & $10.20 \%$ & \\
\hline \multirow{2}{*}{ History of hypertension (\%) } & 65 & 28 & 37 & \multirow{2}{*}{0.023} \\
\hline & $64.36 \%$ & $53.85 \%$ & $75.51 \%$ & \\
\hline \multirow[b]{2}{*}{ Hyperlipidemia (\%) } & 36 & 25 & 11 & \multirow[b]{2}{*}{0.007} \\
\hline & $35.64 \%$ & $48.08 \%$ & $22.45 \%$ & \\
\hline \multirow{2}{*}{ Smoking (\%) } & 22 & 13 & 9 & \multirow{2}{*}{0.419} \\
\hline & $21.78 \%$ & $25.0 \%$ & $18.37 \%$ & \\
\hline \multirow{2}{*}{ Family history of coronary artery disease (\%) } & 16 & 13 & 3 & \multirow{2}{*}{0.009} \\
\hline & $15.84 \%$ & $25.0 \%$ & $6.12 \%$ & \\
\hline \multirow{2}{*}{ Diabetes mellitus (\%) } & 12 & 3 & 9 & \multirow{2}{*}{0.05} \\
\hline & $11.88 \%$ & $5.77 \%$ & $18.37 \%$ & \\
\hline \multirow{2}{*}{ Anxiety/depression (\%) } & 8 & 4 & 4 & \multirow[b]{2}{*}{0.930} \\
\hline & $7.92 \%$ & $7.69 \%$ & $8.16 \%$ & \\
\hline Thyroid disorders (\%) & 24 & 12 & 12 & 0868 \\
\hline Inyroid disorders (\%) & $23.76 \%$ & $23.08 \%$ & $24.49 \%$ & 0.868 \\
\hline Chronic kidney disease (\%) GRACE & 12 & 0 & 12 & \\
\hline Chronic kidney disease (\%) & $11.88 \%$ & $0.0 \%$ & $24.49 \%$ & 0.0001 \\
\hline COPD (\%) & 11 & 3 & 8 & 0088 \\
\hline CUPD (\%) & $10.89 \%$ & $5.77 \%$ & $16.33 \%$ & \\
\hline Diagnostic tests (echocardiography, coronarography, & & & & \\
\hline Left ventricular ejection fraction on admission (\%) & $39.63(9.72)$ & $43.12(9.92)$ & $36.83(9.09)$ & 0.0015 \\
\hline No atherosclerotic changes in coronary arteries (\%) & 42 & 28 & 14 & 0.009 \\
\hline No atnerosclerotic changes in coronary arteries (\%) & $41.58 \%$ & $53.85 \%$ & $28.57 \%$ & 0.009 \\
\hline Insignificant stenoses (\%) & 53 & 23 & 30 & 0.087 \\
\hline molgmincant stenoses (\%) & $52.47 \%$ & $44.23 \%$ & $61.22 \%$ & $0.00 /$ \\
\hline & 6 & 1 & 5 & 0078 \\
\hline Significant stenoses (\%) & $5.94 \%$ & $1.92 \%$ & $10.20 \%$ & 0.078 \\
\hline ECG-ST-segment elevation (\%) ${ }^{\text {GRACE }}$ & 73 & 31 & 42 & 0.003 \\
\hline ECG-S1-segment elevation (\%) & $72.28 \%$ & $59.62 \%$ & $85.71 \%$ & 0.003 \\
\hline QTc on admission (ms) & $468.09(37.70)$ & $461.76(36.57)$ & $479.56(39.37)$ & 0.135 \\
\hline QTc after a few days (ms) & $478.90(82.06)$ & $460.37(99.16)$ & $503.61(46.94)$ & 0.095 \\
\hline Laboratory parameters: & & & & \\
\hline Hemoglobin (mg/dl) & $13.29(1.65)$ & $13.42(1.53)$ & $13.15(1.79)$ & 0.419 \\
\hline Erytrocytes $(\mathrm{x} 106 / \mu \mathrm{l})$ & $4.47(0.65)$ & $4.52(0.57)$ & $4.41(0.72)$ & 0.382 \\
\hline Hematocrit (\%) & $39.37(6.08)$ & $39.31(6.64)$ & $39.31(5.57)$ & 0.924 \\
\hline Leukocytes $(\mathrm{x} 103 / \mu \mathrm{l})$ & $9.89(4.03)$ & $8.36(2.27)$ & $11.52(4.85)$ & $<0.0001$ \\
\hline Glucose on admission (mg/dl) & $126.06(46.06)$ & $107.87(20.14)$ & $145.01(57.27)$ & $<0.0001$ \\
\hline Creatinine $(\mathrm{mg} / \mathrm{dl})^{\text {GRACE }}$ & $0.93(0.46)$ & $0.79(0.25)$ & $1.08(0.59)$ & 0.0013 \\
\hline $\mathrm{eGFR} \operatorname{MDRD}\left(\mathrm{m} / \mathrm{min} / 1.72 \mathrm{~m}^{2}\right)$ & $73.72(27.58)$ & $84.79(25.44)$ & $61.96(25.29)$ & $<0.0001$ \\
\hline CK (IU/l) & $428(908.2)$ & $279.98(311.2)$ & $553.60(1202.51)$ & 0.208 \\
\hline Troponin (significant increase) (\%) GRACE & 88 & 46 & 42 & 0.680 \\
\hline Troponin (significant increase) (\%) & $87.13 \%$ & $88.46 \%$ & $85.71 \%$ & 0.680 \\
\hline Troponin-mean concentration $(\mathrm{ng} / \mathrm{ml})$ & $5.72(11.60)$ & $4.84(6.25)$ & $6.64(15.42)$ & 0.447 \\
\hline Total cholesterol (mg/dl) & $181.2(42.07)$ & $192.07(46.13)$ & $168.76(33.89)$ & 0.009 \\
\hline $\mathrm{LDL}(\mathrm{mg} / \mathrm{dl})$ & $110.3(38.72)$ & $121.35(42.23)$ & $96.65(29.68)$ & 0.003 \\
\hline $\mathrm{HDL}(\mathrm{mg} / \mathrm{dl})$ & $51.65(17.80)$ & $52.31(16.97)$ & $50.83(19.19)$ & 0.709 \\
\hline Triglycerides (mg/dl) & $98.28(52.25)$ & $100.83(54.14)$ & $95.28(51.14)$ & 0.719 \\
\hline CRP $(\mathrm{mg} / \mathrm{l})$ & $40.95(64.92)$ & $16.61(19.73)$ & $59.21(80.48)$ & 0.006 \\
\hline Clinical in-hospital stay and mortality: & & & & \\
\hline RRs on admission ${ }^{\text {GRACE }}$ & $128.8(27.08)$ & $135.31(25.58)$ & $121.94(27.46)$ & 0.012 \\
\hline HR on admission GRACE & $84.39(19.04)$ & $77.54(16.41)$ & $91.65(19.28)$ & 0.0001 \\
\hline Retrosternal chest pain (\%) & 82 & 44 & 38 & 0363 \\
\hline Retrosternal chest pain (\%) & $81.19 \%$ & $84.62 \%$ & $77.55 \%$ & 0.363 \\
\hline
\end{tabular}


TABLE 1: Continued.

\begin{tabular}{|c|c|c|c|c|}
\hline GRACE & $\begin{array}{c}\text { TTS } N=101(\mathrm{SD}) \\
N(\%)\end{array}$ & $\begin{array}{c}\mathrm{TTS} \leq 140 N=52(\mathrm{SD}) \\
N(\%)\end{array}$ & $\begin{array}{c}\mathrm{TTS}>140 N=49(\mathrm{SD}) \\
N(\%)\end{array}$ & $p$ \\
\hline Dyspnea (\%) & $\begin{array}{c}13 \\
12.87 \%\end{array}$ & $\begin{array}{c}4 \\
7.69 \%\end{array}$ & $\begin{array}{c}9 \\
18.37 \%\end{array}$ & 0.109 \\
\hline $\begin{array}{l}\text { Cardiac arrest, pulmonary oedema, cardiogenic shock } \\
(\%)^{\text {GRACE }}\end{array}$ & $\begin{array}{c}18 \\
17.82 \%\end{array}$ & $\begin{array}{c}3 \\
5.77 \%\end{array}$ & $\begin{array}{c}15 \\
30.61 \%\end{array}$ & 0.001 \\
\hline Cardiac rupture (\%) & $\begin{array}{c}3 \\
2.97 \%\end{array}$ & $\begin{array}{c}0 \\
0.0 \%\end{array}$ & $\begin{array}{c}3 \\
6.12 \%\end{array}$ & 0.070 \\
\hline Pneumonia (\%) & $\begin{array}{c}21 \\
20.79 \%\end{array}$ & $\begin{array}{c}4 \\
7.69 \%\end{array}$ & $\begin{array}{c}18 \\
36.73 \%\end{array}$ & 0.0004 \\
\hline Rhythm disturbances (\%) & $\begin{array}{c}11 \\
10.89 \% \\
\end{array}$ & $\begin{array}{c}2 \\
3.85 \% \\
\end{array}$ & $\begin{array}{c}9 \\
17.31 \% \\
\end{array}$ & 0.026 \\
\hline \multicolumn{5}{|l|}{ Pharmacological treatment at discharge } \\
\hline Aspirin & $\begin{array}{c}96 \\
95.05 \%\end{array}$ & $\begin{array}{c}51 \\
98.08 \%\end{array}$ & $\begin{array}{c}45 \\
91.84 \%\end{array}$ & 0.148 \\
\hline ACEI/ARB & $\begin{array}{c}87 \\
86.14 \%\end{array}$ & $\begin{array}{c}47 \\
90.38 \%\end{array}$ & $\begin{array}{c}40 \\
81.63 \%\end{array}$ & 0.203 \\
\hline$\beta$-blockers & $\begin{array}{c}84 \\
83.17 \%\end{array}$ & $\begin{array}{c}47 \\
90.38 \%\end{array}$ & $\begin{array}{c}37 \\
75.51 \%\end{array}$ & 0.046 \\
\hline Statin & $\begin{array}{c}86 \\
85.15 \%\end{array}$ & $\begin{array}{c}48 \\
92.3 \%\end{array}$ & $\begin{array}{c}38 \\
77.55 \%\end{array}$ & 0.037 \\
\hline
\end{tabular}

COPD—chronic obstructive pulmonary disease, ECG-electrocardiogram, eGFR-estimated glomerular filtration rate, CK-creatine kinase, LDL-lowdensity lipoprotein, HDL-high-density lipoprotein, $\mathrm{CRP}$ - c-reactive protein, RR-blood pressure, HR-heart rate, ACEI-angiotensyn converting enzyme inhibitor, and ARB-angiotensin II receptor blocker. ${ }^{*}$ Factors constituting the GRACE scale.

TABle 2: Results-comparison of estimated and observed mortality of 2 Takotsubo groups $(n=101)$.

\begin{tabular}{|c|c|c|c|c|c|c|c|}
\hline & \multicolumn{2}{|c|}{$\begin{array}{c}\text { TTS GRACE points } \\
\text { (medium) }-138.7 \\
N=101\end{array}$} & \multicolumn{2}{|c|}{$\begin{array}{c}\mathrm{TTS} \leq 140 \\
(\text { medium }-108.9) \\
N=52\end{array}$} & \multicolumn{2}{|c|}{$\begin{array}{c}\mathrm{TTS}>140 \\
(\text { medium }-170.2) \\
N=49\end{array}$} & $p$ \\
\hline \multicolumn{8}{|c|}{ GRACE-estimated risk of death (medium) } \\
\hline In-hospital (\%) & & & & & & & 0.004 \\
\hline 6 months $(\%)$ & & & & & & & 0.0004 \\
\hline 1 year $(\%)$ & & & & & & & 0.0001 \\
\hline 3 year $(\%)$ & & & & & & & $<0.0001$ \\
\hline \multicolumn{8}{|c|}{ GRACE-observed mortality } \\
\hline & $n$ & $\%$ & $n$ & $\%$ & $n$ & $\%$ & \\
\hline In-hospital & 6 & 5.94 & 2 & 3.85 & 4 & 8.16 & 0.359 \\
\hline 1 month & 8 & 7.92 & 2 & 3.85 & 6 & 12.24 & 0.118 \\
\hline 3 months & 9 & 8.91 & 2 & 3.85 & 7 & 14.29 & 0.065 \\
\hline 6 months & 11 & 10.89 & 2 & 3.85 & 9 & 18.37 & 0.019 \\
\hline 1 year & 13 & 12.87 & 2 & 3.85 & 11 & 22.45 & 0.005 \\
\hline 3 year & 22 & 21.78 & 2 & 3.85 & 20 & 40.82 & $<0.0001$ \\
\hline 5 year & 23 & 22.77 & 2 & 3.85 & 21 & 42.86 & $<0.0001$ \\
\hline 7 year & 31 & 30.69 & 5 & 9.62 & 26 & 53.06 & $<0.0001$ \\
\hline
\end{tabular}

off value of 153 points, and the sensitivity was $74 \%$, and the specificity was $77 \%$ for TTS (Figure 2).

\section{Discussion}

The first case report on the "apical ballooning cardiomyopathy" was published by Sato et al. [15] almost 30 years ago. Over the years, large international Takotsubo registers have been created, on the basis of which many publications have been developed. Initially, Takotsubo was considered to be a benign clinical entity with good prognosis. Left ventricular wall motion abnormalities generally recover within few weeks, but it was observed that serious life- threatening complications in the form of acute respiratory failure, cardiogenic shock, serious arhytmia, and cardiac rupture may occur in the acute phase of the disease $[1,2]$. There is a greater risk of complications in takotsubo in patients with impaired renal function [16-18]. The pathomechanism of the disease has not yet been fully explained, and the methods to effectively treat those patients to prevent complications in the acute phase and the recurrence of the disease in the follow-up period remain unknown.

Based on the large European Takotsubo registry, the InterTAK score [10] has been developed for the diagnosis of TTS; however, no scale that would assist in the therapeutic 


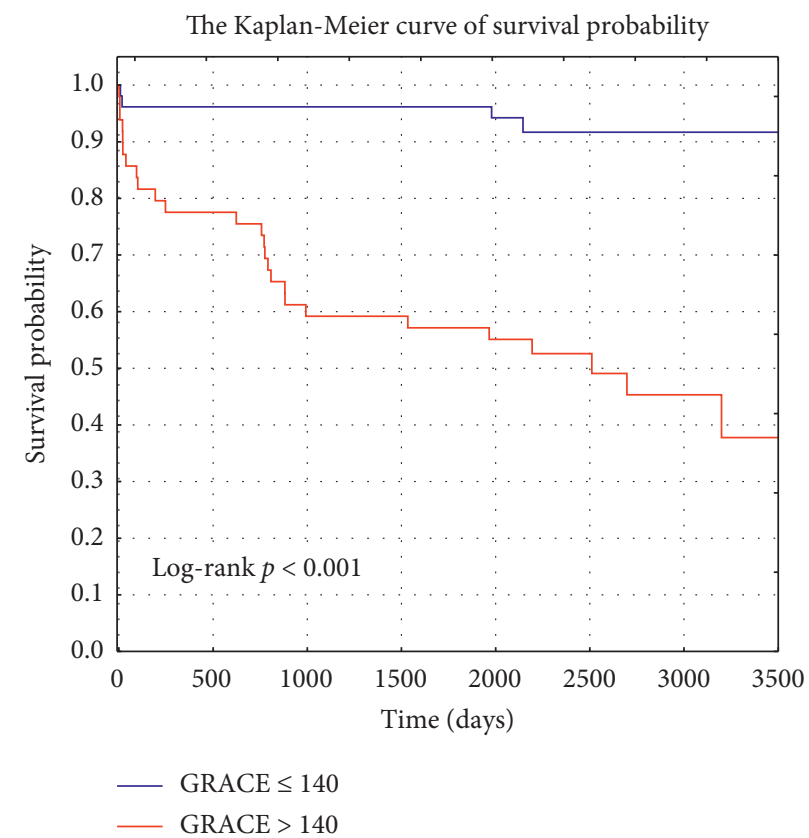

Figure 1: The Kaplan-Meier curve of survival probability of patients with Takotsubo $(n=101)$.

TABLE 3: Univariable and multivariable regression analysis of risk factors of death in Takotsubo $(n=101)$.

\begin{tabular}{|c|c|c|c|c|c|c|}
\hline \multirow{2}{*}{ Predictor } & \multicolumn{3}{|c|}{ Univariable } & \multicolumn{3}{|c|}{ Multivariable } \\
\hline & Odd ratio & $95 \% \mathrm{Cl}^{*}$ & $p$ & Odds ratio & $95 \% \mathrm{Cl}^{*}$ & $p$ \\
\hline Age & 0.942 & $0.904-0.982$ & 0.005 & & & \\
\hline $\mathrm{BMI}<20$ & 1.152 & $1.025-1.294$ & 0.017 & 0.857 & $0.738-0.995$ & 0.042 \\
\hline eGFR MDRD & 1.035 & $1.014-1.056$ & $<0.001$ & 0.954 & $0.920-0.989$ & 0.010 \\
\hline Hypertension & 0.652 & $0.262-1.623$ & 0.357 & & & \\
\hline Smoking & 1.389 & $0.346-5.576$ & 0.643 & & & \\
\hline $\mathrm{EF}<40 \%$ & 1.059 & $1.010-1.110$ & 0.018 & & & \\
\hline Hemoglobin & 1.086 & $0.836-1.411$ & 0.538 & & & \\
\hline CRP & 0.993 & $0.986-1.001$ & 0.104 & & & \\
\hline Troponin & 0.975 & $0.937-1.014$ & 0.208 & & & \\
\hline Creatine kinase & 0.999 & $0.998-1.000$ & 0.051 & & & \\
\hline LDL & 1.024 & $1.006-1.041$ & 0.008 & & & \\
\hline GRACE points & 0.962 & $0.946-0.979$ & $<0.001$ & 1.035 & $1.001-1.069$ & 0.041 \\
\hline
\end{tabular}

Cl-confidence interval.

decision-making and in assessing the prognosis of Takotsubo patients has been developed yet. Considering that the clinical presentation of Takotsubo bears resemblance to acute coronary events, the present study assessed the use of the GRACE scale in the prognosis of TTS patients.

The GRACE scale was developed on the basis of a large international registry of acute coronary syndrome, GRACE (The Global Registry of Acute Coronary Events). Over 102,000 patients with ACS from 250 hospitals in 30 countries were included in this study. The hospital and long-term therapeutic results of patients with acute coronary events were observed, and using these data, the point risk scale was developed [11]. The current GRACE 2.0 scale version is available as an online calculator (GRACE 2.0 ACS Risk Calculator). The European Society of Cardiology has been recommending the use of the
GRACE scale for risk stratification and the selection of appropriate treatment strategy for patients with NSTEMI since $2010[19,20]$.

Santoro et al. demonstrated the value of persistent STsegment elevation in electrocardiography as a predictor of complications during hospitalization and adverse events in an observational study [21]. Takotsubo patients included in the Italian-German GEIST registry were classified as low-risk and high-risk patients based on 4 variables that were identified as independent predictors of hospital complications and adverse events in follow-up (male gender, history of neurological disorders, right ventricular involvement, and left ventricular ejection fraction (LVEF) [22].

In a group of 537 Takotsubo patients, Scudiero et al. [23] noted a significantly higher mortality in the group of patients with a GRACE score > $140(17 \%$ vs $8 \%)$ after an average 


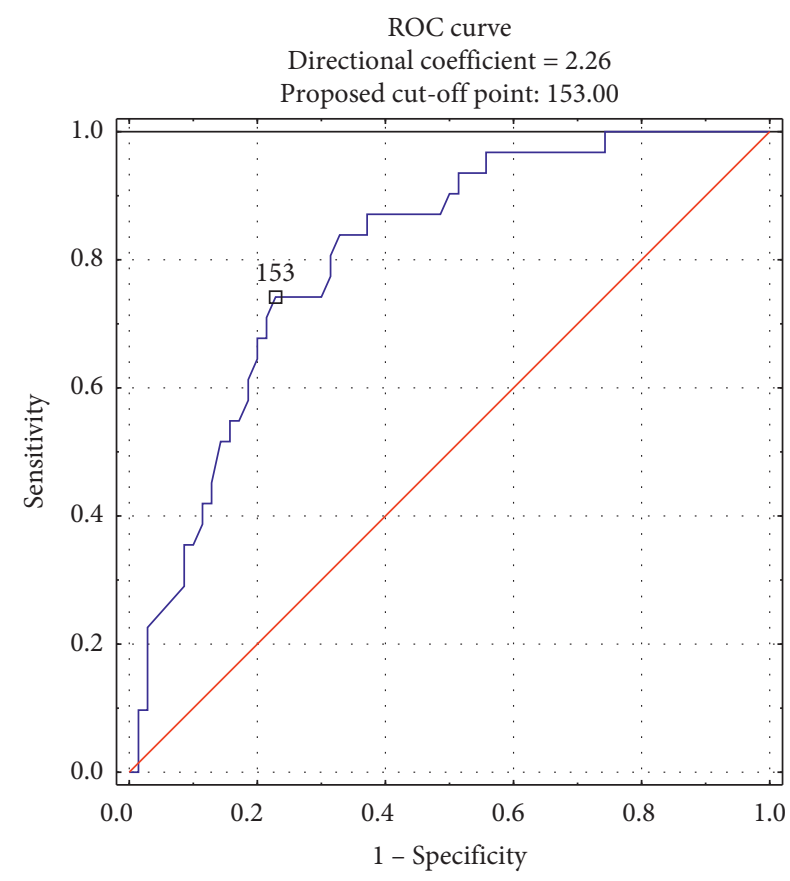

FIGURE 2: Receiver operating characteristic (ROC) curves for GRACE risk score in the prediction of 7-year mortality in patients with Takotsubo syndrome 1 -Specificity.

follow-up period of 40 months. In the presented analysis, the differences in the deaths rates of patients with a GRACE score $>140$ and $\leq 140$ in a similar 3-year follow-up period were even higher, with the mortality rate amounting to $40.8 \%$ vs $3.85 \%$. In addition, in a group of 561 TTS patients, Scudiero et al. [24] determined a significant discriminatory value of the GRACE score for the prognostication of mortality and cerebrovascular incidents after an average follow-up period of 29 months. For all-cause mortality, the area under ROC curve (AUC) was calculated as 0.67 (95\% CI $0.60-0.73 ;<0.001)$. In this paper, the discriminatory value of the GRACE scale was even stronger (AUC-0.805, 95\% CI $0.718-0.892,<0.001)$, which is the result of the high mortality of our patients with a higher GRACE score and a longer observation period.

In addition, the applicability of the CHA2DS2-VASc scale, which is used to assess the thromboembolic risk in patients with atrial fibrillation, was evaluated for TTS. In a group of 371 patients, Parodi et al. [25] determined the significant discriminatory ability of the CHA2DS2-VASc scale for the prediction of mortality rate and cardiac and cerebrovascular events. At the same time, a comparison of both scales in the Takotsubo group demonstrated the advantage of the GRACE scale in predicting the prognosis of TTS patients.

It is beyond any doubt that a scale is needed for the assessment of and treatment qualification in the case of Takotsubo syndrome, as well as other acute cardiologic entities. However, until a new specific scale dedicated to TTS will be developed and validated, the GRACE score could be useful for the TTS management. Further research assessing the usefulness of the GRACE scale in the assessment of prognosis of Takotsubo patients is needed to more precise estimation of death risk in these patients in long-term follow-up. Adaptation of the scale to TTS may facilitate the treatment decision-making for Takotsubo patients and in particular the decision on the minimum time of hospitalization, including observation in the intensive cardiology care unit.

\section{Conclusions}

The GRACE scale is highly valuable for the prognostication of death risk in patients with TTS in the early and long-term follow-up period. Further analyses of the application of GRACE scale and other scales used for the assessment of potential risks should be performed on more extensive groups of Takotsubo patients.

5.1. Study Limitation. The greatest study limitation is the inclusion of a low number of TTS patients in the study. Another limitation is that the analysis is retrospective in nature. In the observational study carried out on the PESEL database, only the information on the death dates of the patients was obtained, but the death causes of the deaths were unknown.

\section{Data Availability}

The clinical data used to support the findings of this study are included within the article. The particular data are available from the corresponding author upon request.

\section{Conflicts of Interest}

The authors declare no conflicts of interest.

\section{Acknowledgments}

The authors would like to express our thanks to Prof. Anna Tomaszuk-Kazberuk, Dr Paweł Drozdowski, Dr Jerzy Bychowski, and Dr Romuald Krynicki for providing the medical records of Takotsubo patients.

\section{References}

[1] J.-R. Ghadri, I. S. Wittstein, A. Prasad et al., "International expert consensus document on Takotsubo syndrome (part I): clinical characteristics, diagnostic criteria, and pathophysiology," European Heart Journal, vol. 39, no. 22, pp. 20322046, 2018.

[2] J.-R. Ghadri, I. S. Wittstein, A. Prasad et al., "International expert consensus document on Takotsubo syndrome (part II): diagnostic workup, outcome, and management," European Heart Journal, vol. 39, no. 22, pp. 2047-2062, 2018.

[3] M. Zalewska-Adamiec, H. Bachorzewska-Gajewska, A. Tomaszuk-Kazberuk et al., "Takotsubo cardiomyopathy: serious early complications and two-year mortality-a 101 case study," Netherlands Heart Journal, vol. 24, no. 9, pp. 511-519, 2016.

[4] C. Templin, J. R. Ghadri, J. Diekmann et al., "Clinical features and outcomes of Takotsubo (stress) cardiomyopathy," The New England Journal of Medicine, vol. 373, no. 373, pp. 929-938, 2015. 
[5] M. Zalewska-Adamiec, H. Bachórzewska-Gajewska, P. Kralisz, M. Tałałaj, M. Pryzmont, and S. Dobrzycki, "Sudden cardiac arrest in the course of Takotsubo syndrome in a 15-year-old girl," Advances in Interventional Cardiology, vol. 14, no. 3, pp. 318-319, 2018.

[6] M. Zalewska-Adamiec, H. Bachórzewska-Gajewska, M. Kożuch, M. Frank, T. Hirnle, and S. Dobrzycki, "Cardiac rupture in Takotsubo cardiomyopathy treated surgically," Advances in Interventional Cardiology, vol. 3, no. 3, pp. 278-279, 2016.

[7] V. Farooq, D. van Klaveren, E. W. Steyerberg et al., "Anatomical and clinical characteristics to guide decision making between coronary artery bypass surgery and percutaneous coronary intervention for individual patients: development and validation of SYNTAX score II," The Lancet, vol. 381, no. 9867, pp. 639-650, 2013.

[8] K. A. Fox, G. Fitzgerald, E. Puymirat et al., "Should patients with acute coronary disease be stratified for management according to their risk? Derivation, external validation and outcomes using the updated GRACE risk score," BMJ Open, vol. 4, Article ID e004425, 2014.

[9] H. A. van den Ham, O. H. Klungel, D. E. Singer, H. G. M. Leufkens, and T. P. van Staa, "Comparative performance of ATRIA, CHADS2, and CHA2DS2-VASc risk scores predicting stroke in patients with atrial fibrillation," Journal of the American College of Cardiology, vol. 66, no. 17, pp. 1851-1859, 2015.

[10] J. R. Ghadri, V. L. Cammann, S. Jurisic et al., "A novel clinical score (InterTAK diagnostic score) to differentiate Takotsubo syndrome from acute coronary syndrome: results from the International Takotsubo Registry," European Journal of Heart Failure, vol. 19, no. 8, pp. 1036-1042, 2017.

[11] M. Roffi, C. Patrono, J.-P. Collet et al., "2015 ESC guidelines for the management of acute coronary syndromes in patients presenting without persistent ST-segment elevation," European Heart Journal, vol. 37, no. 3, pp. 267-315, 2016.

[12] F. J. Neumann, M. Sousa-Uva, A. Ahlsson et al., "2018 ESC/ EACTS guidelines on myocardial revascularization," EuroIntervention, vol. 14, no. 14, pp. 1435-1534, 2019.

[13] D. C. Scantlebury and A. Prasad, "Diagnosis of Takotsubo cardiomyopathy," Circulation Journal, vol. 78, no. 9, pp. 2129-2139, 2014.

[14] A. R. Lyon, E. Bossone, B. Schneider et al., "Current state of knowledge on Takotsubo syndrome: a position statement from the taskforce on Takotsubo syndrome of the heart failure association of the European society of cardiology," European Journal of Heart Failure, vol. 18, no. 1, pp. 8-27, 2016.

[15] H. Sato, H. Tateishi, T. Uchida et al., "Tako-tsubo-like left ventricular dysfunction due to multivessel coronary spasm," in Clinical Aspect of Myocardial Injury: From Ischemia to Heart Failure, pp. 56-64, Kagakuhyoronsha Publishing, Tokyo, Japan, 1990.

[16] F. Santoro, A. Ferraretti, R. Ieva et al., "Renal impairment and outcome in patients with Takotsubo cardiomyopathy," The American Journal of Emergency Medicine, vol. 34, no. 3, pp. 548-552, 2016.

[17] K. Ando, H. Sukekawa, A. Takahata et al., "Renal dysfunction indicative of outcomes in hospitalized patients with takotsubo syndrome," European Heart Journal: Acute Cardiovascular Care, vol. 7, no. 8, pp. 723-731, 2018.

[18] M. Zalewska-Adamiec, J. Małyszko, H. BachórzewskaGajewska et al., "Takotsubo syndrome and chronic kidney disease-deadly duet in long-term follow-up," Polish Archives of Internal Medicine, vol. 128, no. 9, pp. 518-523, 2018.
[19] C. W. Hamm, J. P. Bassand, S. Agewall et al., "ESC guidelines for the management of acute coronary syndromes in patients presenting without persistent ST-segment elevation: the task force for the management of acute coronary syndromes (ACS) in patients presenting without persistent ST-segment elevation of the European Society of Cardiology (ESC)," European Heart Journal, vol. 32, no. 23, pp. 2999-3054, 2011.

[20] S. Windecker, P. Kolh, F. Alfonso et al., "2014 ESC/EACTS guidelines on myocardial revascularization: the task force on myocardial revascularization of the European Society of Cardiology (ESC) and the European Association for CardioThoracic Surgery (EACTS) developed with the special contribution of the European Association of Percutaneous Cardiovascular Interventions (EAPCI)," European Heart Journal, vol. 35, no. 37, pp. 2541-2619, 2014.

[21] F. Santoro, T. Stiermaier, N. Tarantino et al., "Impact of persistent ST elevation on outcome in patients with Takotsubo syndrome. Results from the German Italian Stress Cardiomyopathy (GEIST) registry," International Journal of Cardiology, vol. 255, pp. 140-144, 2018.

[22] F. Santoro, I. J. Núñez Gil, T. Stiermaier et al., "Assessment of the German and Italian stress cardiomyopathy score for risk stratification for in-hospital complications in patients with Takotsubo syndrome," JAMA Cardiology, vol. 4, no. 9, pp. 892-899, 2019.

[23] F. Scudiero, L. Arcari, B. Bacchi et al., "Discriminatory ability of GRACE risk score to predict outcomes in patients with Takotsubo syndrome," European Heart Journal, vol. 39, p. 248, 2018.

[24] F. Scudiero, L. Arcari, L. Cacciotti et al., "Prognostic relevance of GRACE risk score in Takotsubo syndrome," European Heart Journal: Acute Cardiovascular Care, Article ID, 204887261988236, 2019.

[25] G. Parodi, F. Scudiero, R. Citro et al., "Risk stratification using the CHA2DS2-VASc score in Takotsubo syndrome: data from the Takotsubo Italian network," Journal of the American Heart Association, vol. 6, no. 9, Article ID e006065, 2017. 